\title{
BIFURCATION AND MULTIPLICITY RESULTS FOR CLASSES OF $p, q$-LAPLACIAN SYSTEMS
}

\author{
Ratnasingham Shivaji — Byunguae Son
}

Abstract. We study positive solutions to boundary value problems of the form

$$
\begin{cases}-\Delta_{p} u=\lambda\left\{u^{p-1-\alpha}+f(v)\right\} & \text { in } \Omega, \\ -\Delta_{q} v=\lambda\left\{v^{q-1-\beta}+g(u)\right\} & \text { in } \Omega, \\ u=0=v & \text { on } \partial \Omega,\end{cases}
$$

where $\Delta_{m} u:=\operatorname{div}\left(|\nabla u|^{m-2} \nabla u\right), m>1$, is the $m$-Laplacian operator of $u, \lambda>0, p, q>1, \alpha \in(0, p-1), \beta \in(0, q-1)$ and $\Omega$ is a bounded domain in $\mathbb{R}^{N}, N \geq 1$, with smooth boundary $\partial \Omega$. Here $f, g:[0, \infty) \rightarrow \mathbb{R}$ are nondecreasing continuous functions with $f(0)=0=g(0)$. We first establish that for $\lambda \approx 0$ there exist positive solutions bifurcating from the trivial branch $(\lambda, u \equiv 0, v \equiv 0)$ at $(0,0,0)$. We further discuss an existence result for all $\lambda>0$ and a multiplicity result for a certain range of $\lambda$ under additional assumptions on $f$ and $g$. We employ the method of sub-super solutions to establish our results.

\section{Introduction}

Consider boundary value problems of the form

$$
\begin{cases}-\Delta_{p} u=\lambda \tilde{f}(u) & \text { in } \Omega, \\ u=0 & \text { on } \partial \Omega\end{cases}
$$

2010 Mathematics Subject Classification. 35J25, 35J57, 35J60.

Key words and phrases. Positive solutions; bifurcation; existence; multiplicity.

This work was partially supported by a grant from the Simons Foundation (\# 317872) to Ratnasingham Shivaji. 
where $\Delta_{p} u:=\operatorname{div}\left(|\nabla u|^{p-2} \nabla u\right), p>1$, is the $p$-Laplacian operator of $u, \lambda>0$ and $\Omega$ is a bounded domain in $\mathbb{R}^{N}, N \geq 1$, with smooth boundary $\partial \Omega$. Here $\tilde{f}:[0, \infty) \rightarrow \mathbb{R}$ is a nondecreasing continuous function. When $\tilde{f}(0)>0$, there is a rich history on the study of positive solutions. The authors in [5] have considered such problems in the Laplacian case $(p=2)$ and established an existence result for all $\lambda>0$ and a multiplicity result for a certain range of $\lambda$ under additional assumptions on $\widetilde{f}$. Later in [7], these results were extended to the $p$-Laplacian case $(p>1)$. In particular, the authors in [7] proved the existence of a positive solution for all $\lambda>0$ when $\widetilde{f}$ is $p$ sublinear at $\infty$, and multiplicity results for a certain range of $\lambda$ when there exist $a$ and $b$ such that $0<a<b$ and $\left(a^{p-1} / \widetilde{f}(a)\right) /\left(b^{p-1} / \widetilde{f}(b)\right)$ is sufficiently large. See also [1], [2] and [6] for related results in the case $\widetilde{f}(0)>0$. Here, we focus on the case $\widetilde{f}(0)=0$. If $\widetilde{f}(0)>0$, then $u \equiv 0$ is a very useful nonnegative strict subsolution to help with the study of establishing positive solutions. In this paper, $u \equiv 0$ is a solution for each $\lambda>0$ and hence we lack the presence of this trivial nonnegative strict subsolution. However, we use the presence of the term $u^{p-1-\alpha}$ as our advantage to overcome this difficulty and show that positive solutions bifurcate at $(0,0)$ from the trivial branch $(\lambda, u \equiv 0)$. Under additional properties on $\widetilde{f}$, we establish further existence and multiplicity results. We also extend these results to classes of $p, q$-Laplacian systems. In particular, we consider boundary value problems of the form

$$
\begin{cases}-\Delta_{p} u=\lambda\left\{u^{p-1-\alpha}+f(v)\right\} & \text { in } \Omega, \\ -\Delta_{q} v=\lambda\left\{v^{q-1-\beta}+g(u)\right\} & \text { in } \Omega, \\ u=0=v & \text { on } \partial \Omega,\end{cases}
$$

where $\Delta_{m} u:=\operatorname{div}\left(|\nabla u|^{m-2} \nabla u\right), m>1$, is the $m$-Laplacian operator of $u$, $\lambda>0, p, q>1, \alpha \in(0, p-1), \beta \in(0, q-1)$ are parameters and $\Omega$ is a bounded domain in $\mathbb{R}^{N}, N \geq 1$, with smooth boundary $\partial \Omega$. Here $f, g:[0, \infty) \rightarrow \mathbb{R}$ are nondecreasing continuous functions with $f(0)=0=g(0)$. Clearly for all $\lambda$, $(u \equiv 0, v \equiv 0)$ is a solution of (1.1). In this paper, we are interested in the study of solution $(u, v) \in W^{1, p}(\Omega) \cap C(\bar{\Omega}) \times W^{1, q}(\Omega) \cap C(\bar{\Omega})$ with $u, v>0$ in $\Omega$. We first establish:

THeorem 1.1. There exists $\lambda_{0}>0$ such that for all $\lambda \in\left(0, \lambda_{0}\right)$, (1.1) has a positive solution $(u, v)$ such that $\|u\|_{\infty} \rightarrow 0,\|v\|_{\infty} \rightarrow 0$ as $\lambda \rightarrow 0$ (see Figure 1).

Next we consider the case when $f, g$ satisfy the following combined $p, q$ sublinear condition at $\infty$ :

$$
\left(\mathrm{H}_{1}\right) \lim _{s \rightarrow \infty} \frac{f\left(M g(s)^{1 /(q-1)}\right)}{s^{p-1}}=0, \text { for all } M>0,
$$
and establish: 
Bifurcation and Multiplicity Results for Classes of $p, q$-Laplacian Systems 105



FIGURE 1

THEOREM 1.2. Let $\left(\mathrm{H}_{1}\right)$ hold. Then (1.1) has a positive solution $(u, v)$ for all $\lambda>0$ (see Figure 2).

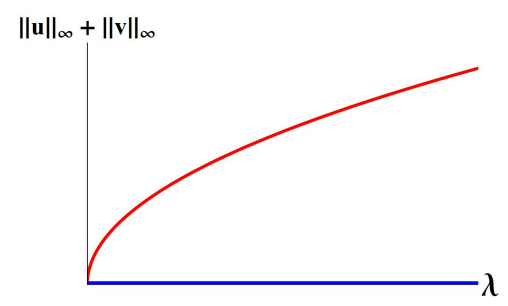

Figure 2

If in addition, $f, g$ satisfy:

$\left(\mathrm{H}_{2}\right)$ There exist positive constants $a_{1}$ and $a_{2}\left(>a_{1}\right)$ such that

$$
\frac{\min \left[\min \left\{a_{1}^{\alpha}, a_{1}^{p-1} / f\left(a_{1}\right)\right\}, \min \left\{a_{1}^{\beta}, a_{1}^{q-1} / g\left(a_{1}\right)\right\}\right]}{\max \left\{a_{2}^{p-1} / f\left(a_{2}\right), a_{2}^{q-1} / g\left(a_{2}\right)\right\}} \geq C(\Omega),
$$

where

$$
C(\Omega)=2 \max \left\{\left\|e_{p}\right\|_{\infty}^{p-1},\left\|e_{q}\right\|_{\infty}^{q-1}\right\} \min \left[\max \left\{A_{p, p}, A_{p, q}\right\}, \max \left\{A_{q, p}, A_{q, q}\right\}\right],
$$

$e_{m}$ is the solution of $-\Delta_{m} e=1 ; \Omega, e=0 ; \partial \Omega$,

$$
A_{m, n}=\frac{(N+m-1)^{N+n-1}}{R^{n} N^{N-1}(m-1)^{n-1}}
$$

and $R$ is the radius of the largest inscribed ball $B_{R}$ in $\Omega$, we prove:

Theorem 1.3. Let $\left(\mathrm{H}_{1}\right)-\left(\mathrm{H}_{2}\right)$ hold. Then (1.1) has at least three positive solutions for $\lambda \in\left(\lambda_{*}, \lambda^{*}\right)$, where (see Figure 3 )

$$
\begin{aligned}
& \lambda_{*}=\min \left[\max \left\{\frac{a_{2}^{p-1}}{f\left(a_{2}\right)} A_{p, p}, \frac{a_{2}^{q-1}}{g\left(a_{2}\right)} A_{p, q}\right\}, \max \left\{\frac{a_{2}^{p-1}}{f\left(a_{2}\right)} A_{q, p}, \frac{a_{2}^{q-1}}{g\left(a_{2}\right)} A_{q, q}\right\}\right], \\
& \lambda^{*}=\min \left[\frac{1}{2\left\|e_{p}\right\|_{\infty}^{p-1}} \min \left\{a_{1}^{\alpha}, \frac{a_{1}^{p-1}}{f\left(a_{1}\right)}\right\}, \frac{1}{2\left\|e_{q}\right\|_{\infty}^{q-1}} \min \left\{a_{1}^{\beta}, \frac{a_{1}^{q-1}}{g\left(a_{1}\right)}\right\}\right] .
\end{aligned}
$$




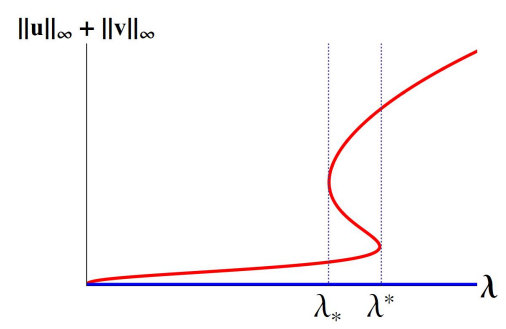

FIGURE 3

In the case of single equations, namely equation of the form

$$
\begin{cases}-\Delta_{p} u=\lambda\left\{u^{p-1-\alpha}+f(u)\right\} & \text { in } \Omega, \\ u=0 & \text { on } \partial \Omega\end{cases}
$$

our results easily reduce to:

THEOREM 1.4. There exists $\lambda_{0}>0$ such that for all $\lambda \in\left(0, \lambda_{0}\right),(1.2)$ has a positive solution $u$ such that $\|u\|_{\infty} \rightarrow 0$ as $\lambda \rightarrow 0$.

TheOREm 1.5. Assume $f(s) / s^{p-1} \rightarrow 0$ as $s \rightarrow \infty$. Then (1.2) has a positive solution $u$ for all $\lambda>0$.

TheOREM 1.6. Assume $f(s) / s^{p-1} \rightarrow 0$ as $s \rightarrow \infty$ and there exist positive constants $a_{1}$ and $a_{2}\left(>a_{1}\right)$ such that

$$
\frac{\min \left\{a_{1}^{\alpha}, a_{1}^{p-1} / f\left(a_{1}\right)\right\}}{a_{2}^{p-1} / f\left(a_{2}\right)}>\widetilde{C}(\Omega), \quad \text { where } \widetilde{C}(\Omega)=2\left\|e_{p}\right\|_{\infty}^{p-1} A_{p, p},
$$

$e_{p}$ is a solution of $-\Delta_{p} e=1 ; \Omega, e=0 ; \partial \Omega$,

$$
A_{p, p}=\frac{(N+p-1)^{N+p-1}}{R^{p} N^{N-1}(p-1)^{p-1}}
$$

and $R$ is the radius of the largest inscribed ball $B_{R}$ in $\Omega$. Then (1.2) has a positive solution for all $\lambda$, and at least three positive solutions for $\lambda \in\left(\lambda_{*}, \lambda^{*}\right)$, where

$$
\lambda_{*}=\frac{a_{2}^{p-1}}{f\left(a_{2}\right)} A_{p, p}, \quad \lambda^{*}=\frac{1}{2\left\|e_{p}\right\|_{\infty}^{p-1}} \min \left\{a_{1}^{\alpha}, \frac{a_{1}^{p-1}}{f\left(a_{1}\right)}\right\} .
$$

REMARK 1.7. If $s^{p-1} /\left(s^{p-1-\alpha}+f(s)\right)$ is strictly increasing on $(0, \infty)$, which will be the case if $s^{p-1} / f(s)$ is increasing on $(0, \infty)$, and there exists $c>0$ such that $s^{p-1-\alpha}+f(s) \leq c\left(s^{p-1}+1\right)$ for $s \in[0, \infty)$, which will be satisfied if $f(s) / s^{p-1} \rightarrow 0$ as $s \rightarrow \infty$, then (1.2) has at most one positive solution for all $\lambda>0$ (see [4]). Note that the hypotheses in Theorem 1.6 do not allow $s^{p-1} / f(s)$ to be increasing for all $s \in(0, \infty)$. 
We establish Theorems 1.1-1.3 by the method of sub-super solutions. By a subsolution of (1.1) we mean a pair of functions $(\psi, \bar{\psi}) \in W^{1, p}(\Omega) \cap C(\bar{\Omega}) \times$ $W^{1, q}(\Omega) \cap C(\bar{\Omega})$ such that $(\psi, \bar{\psi})=(0,0)$ on $\partial \Omega$ and

$$
\begin{array}{ll}
\int_{\Omega}|\nabla \psi|^{p-2} \nabla \psi \cdot \nabla \zeta \leq \int_{\Omega} \lambda\left(\psi^{p-1-\alpha}+f(\bar{\psi})\right) \zeta & \text { for all } \zeta \in W, \\
\int_{\Omega}|\nabla \bar{\psi}|^{q-2} \nabla \bar{\psi} \cdot \nabla \zeta \leq \int_{\Omega} \lambda\left(\bar{\psi}^{q-1-\beta}+g(\psi)\right) \zeta \quad \text { for all } \zeta \in W,
\end{array}
$$

where $W=\left\{h \in C_{0}^{\infty}(\Omega): h \geq 0\right.$ in $\left.\Omega\right\}$. By a supersolution of (1.1) we mean a pair of functions $(\phi, \bar{\phi}) \in W^{1, p}(\Omega) \cap C(\bar{\Omega}) \times W^{1, q}(\Omega) \cap C(\bar{\Omega})$ such that $(\phi, \bar{\phi})=$ $(0,0)$ on $\partial \Omega$ and

$$
\begin{array}{ll}
\int_{\Omega}|\nabla \phi|^{p-2} \nabla \phi \cdot \nabla \zeta \geq \int_{\Omega} \lambda\left(\phi^{p-1-\alpha}+f(\bar{\phi})\right) \zeta & \text { for all } \zeta \in W, \\
\int_{\Omega}|\nabla \bar{\phi}|^{q-2} \nabla \bar{\phi} \cdot \nabla \zeta \geq \int_{\Omega} \lambda\left(\bar{\phi}^{q-1-\beta}+g(\phi)\right) \zeta & \text { for all } \zeta \in W .
\end{array}
$$

By a strict subsolution of (1.1) we mean a subsolution which is not a solution. By a strict supersolution of (1.1) we mean a supersolution which is not a solution. Then the following results are well-known (see [3], [6] and [8]).

Proposition 1.8. If there exist a subsolution $(\psi, \bar{\psi})$ and a supersolution $(\phi, \bar{\phi})$ of (1.1) such that $(\psi, \bar{\psi}) \leq(\phi, \bar{\phi})$, then (1.1) has at least one solution $(u, v) \in W^{1, p}(\Omega) \cap C(\bar{\Omega}) \times W^{1, q}(\Omega) \cap C(\bar{\Omega})$ satisfying $(\psi, \bar{\psi}) \leq(u, v) \leq(\phi, \bar{\phi})$.

Proposition 1.9. Let $f$ and $g$ be nonnegative and nondecreasing, and suppose there exist a subsolution $\left(\psi_{1}, \bar{\psi}_{1}\right)$, a strict supersolution $\left(\phi_{1}, \bar{\phi}_{1}\right)$, a strict subsolution $\left(\psi_{2}, \bar{\psi}_{2}\right)$, and a supersolution $\left(\phi_{2}, \bar{\phi}_{2}\right)$ for $(1.1)$ such that $\left(\psi_{1}, \bar{\psi}_{1}\right) \leq$ $\left(\phi_{1}, \bar{\phi}_{1}\right) \leq\left(\phi_{2}, \bar{\phi}_{2}\right),\left(\psi_{1}, \bar{\psi}_{1}\right) \leq\left(\psi_{2}, \bar{\psi}_{2}\right) \leq\left(\phi_{2}, \bar{\phi}_{2}\right)$, and $\left(\psi_{2}, \bar{\psi}_{2}\right) \not \leq\left(\phi_{1}, \bar{\phi}_{1}\right)$. Then (1.1) has at least three distinct solutions $\left(u_{i}, v_{i}\right), i=1,2,3$, such that

$$
\begin{gathered}
\left(u_{1}, v_{1}\right) \in\left[\left(\psi_{1}, \bar{\psi}_{1}\right),\left(\phi_{1}, \bar{\phi}_{1}\right)\right], \quad\left(u_{2}, v_{2}\right) \in\left[\left(\psi_{2}, \bar{\psi}_{2}\right),\left(\phi_{2}, \bar{\phi}_{2}\right)\right], \\
\left(u_{3}, v_{3}\right) \in\left[\left(\psi_{1}, \bar{\psi}_{1}\right),\left(\phi_{2}, \bar{\phi}_{2}\right)\right] \backslash\left(\left[\left(\psi_{1}, \bar{\psi}_{1}\right),\left(\phi_{1}, \bar{\phi}_{1}\right)\right] \cup\left[\left(\psi_{2}, \bar{\psi}_{2}\right),\left(\phi_{2}, \bar{\phi}_{2}\right)\right]\right) .
\end{gathered}
$$

We will establish Theorem 1.1 in Section 2 and Theorems 1.2-1.3 in Section 3. Finally in Section 4, we discuss simple examples satisfying the hypotheses of Theorems 1.3 and 1.6.

\section{Proof of Theorem 1.1}

Proof of Theorem 1.1. Let $\gamma>0$ be such that $\gamma \alpha<1, \gamma \beta<1, \gamma(p-1)<$ 1 and $\gamma(q-1)<1$. For sufficiently small $\lambda$, we have

$$
\begin{aligned}
& 1 \geq \lambda^{1-\gamma \alpha}\left\|e_{p}\right\|_{\infty}^{p-1-\alpha}+\lambda^{1-\gamma(p-1)} f\left(\lambda^{\gamma}\left\|e_{q}\right\|_{\infty}\right), \\
& 1 \geq \lambda^{1-\gamma \beta}\left\|e_{q}\right\|_{\infty}^{q-1-\beta}+\lambda^{1-\gamma(q-1)} g\left(\lambda^{\gamma}\left\|e_{p}\right\|_{\infty}\right) .
\end{aligned}
$$


Let $\left(w_{1}, \bar{w}_{1}\right)=\left(\lambda^{\gamma} e_{p}, \lambda^{\gamma} e_{q}\right)$. Then

$$
\begin{aligned}
-\Delta_{p} w_{1} & =\lambda^{\gamma(p-1)} \geq \lambda\left(\left(\lambda^{\gamma}\left\|e_{p}\right\|_{\infty}\right)^{p-1-\alpha}+f\left(\lambda^{\gamma}\left\|e_{q}\right\|_{\infty}\right)\right) \\
& \geq \lambda\left(\left(\lambda^{\gamma} e_{p}\right)^{p-1-\alpha}+f\left(\lambda^{\gamma} e_{q}\right)\right)=\lambda\left(w_{1}^{p-1-\alpha}+f\left(\bar{w}_{1}\right)\right), \\
-\Delta_{q} \bar{w}_{1} & =\lambda^{\gamma(q-1)} \geq \lambda\left(\left(\lambda^{\gamma}\left\|e_{q}\right\|_{\infty}\right)^{q-1-\beta}+g\left(\lambda^{\gamma}\left\|e_{p}\right\|_{\infty}\right)\right) \\
& \geq \lambda\left(\left(\lambda^{\gamma} e_{q}\right)^{q-1-\beta}+g\left(\lambda^{\gamma} e_{p}\right)\right)=\lambda\left(\bar{w}_{1}^{q-1-\beta}+g\left(w_{1}\right)\right) .
\end{aligned}
$$

Thus, $\left(w_{1}, \bar{w}_{1}\right)$ is a supersolution of (1.1) for sufficiently small $\lambda$. Next, we construct a positive subsolution of (1.1). Let $z_{m}>0 ; \Omega$ be the eigenfunction with $\left\|z_{m}\right\|_{\infty}=1$ corresponding the principal eigenvalue $\lambda_{1, m}$ of the problem

$$
\begin{cases}-\Delta_{m} z=\lambda|z|^{m-2} z & \text { in } \Omega, \\ z=0 & \text { on } \partial \Omega .\end{cases}
$$

Let $m_{\lambda}>0$ be sufficiently small such that $\lambda_{1, p} m_{\lambda}^{\alpha} \leq \lambda$ and $\lambda_{1, q} m_{\lambda}^{\beta} \leq \lambda$. Let $\left(\psi_{1}, \bar{\psi}_{1}\right)=\left(m_{\lambda} z_{p}, m_{\lambda} z_{q}\right)$. Then

$$
\begin{aligned}
& -\Delta_{p} \psi_{1}=\lambda_{1, p}\left(m_{\lambda} z_{p}\right)^{p-1} \leq \lambda\left(m_{\lambda} z_{p}\right)^{p-1-\alpha} \leq \lambda\left(\psi_{1}^{p-1-\alpha}+f\left(\bar{\psi}_{1}\right)\right), \\
& -\Delta_{q} \bar{\psi}_{1}=\lambda_{1, q}\left(m_{\lambda} z_{q}\right)^{q-1} \leq \lambda\left(m_{\lambda} z_{q}\right)^{q-1-\beta} \leq \lambda\left(\bar{\psi}_{1}^{q-1-\beta}+g\left(\psi_{1}\right)\right) .
\end{aligned}
$$

Thus, $\left(\psi_{1}, \bar{\psi}_{1}\right)$ is a subsolution of (1.1) for all $\lambda>0$. Further, we can choose $m_{\lambda}$ sufficiently small such that $\left(\psi_{1}, \bar{\psi}_{1}\right) \leq\left(w_{1}, \bar{w}_{1}\right)$. By Proposition 1.8, there exists a positive solution $(u, v)$ such that $\left(\psi_{1}, \bar{\psi}_{1}\right) \leq(u, v) \leq\left(w_{1}, \bar{w}_{1}\right)$ for sufficiently small $\lambda$, and note that $\left\|w_{1}\right\|_{\infty} \rightarrow 0$ and $\left\|\bar{w}_{1}\right\|_{\infty} \rightarrow 0$ as $\lambda \rightarrow 0$.

\section{Proofs of Theorems 1.2 and 1.3}

Proof of Theorem 1.2. Let $\left(\psi_{1}, \bar{\psi}_{1}\right)$ be as before in the proof of Theorem 1.1. Then $\left(\psi_{1}, \bar{\psi}_{1}\right)$ is a subsolution for all $\lambda>0$. Next, we construct a positive supersolution $\left(\phi_{1}, \bar{\phi}_{1}\right)$ of (1.1). If $f$ and $g$ are bounded, choose $M_{\lambda} \gg 1$ such that $M_{\lambda}^{\alpha} \geq 2 \lambda^{(p-1-\alpha) /(p-1)}\left\|e_{p}\right\|_{\infty}^{p-1-\alpha}, M_{\lambda}^{\beta} \geq 2 \lambda^{(q-1-\beta) /(q-1)}\left\|e_{q}\right\|_{\infty}^{q-1-\beta}$, $M_{\lambda}^{p-1} \geq 2\|f\|_{\infty}$ and $M_{\lambda}^{q-1} \geq 2\|g\|_{\infty}$.

Let $\left(\phi_{1}, \bar{\phi}_{1}\right)=\left(\lambda^{1 /(p-1)} M_{\lambda} e_{p}, \lambda^{1 /(q-1)} M_{\lambda} e_{q}\right)$. Then

$$
\begin{aligned}
-\Delta_{p} \phi_{1} & =\lambda M_{\lambda}^{p-1} \geq \lambda\left(\lambda^{(p-1-\alpha) /(p-1)} M_{\lambda}^{p-1-\alpha}\left\|e_{p}\right\|_{\infty}^{p-1-\alpha}+\|f\|_{\infty}\right) \\
& \geq \lambda\left(\left(\lambda^{1 /(p-1)} M_{\lambda} e_{p}\right)^{p-1-\alpha}+f\left(\lambda^{1 /(q-1)} M_{\lambda} e_{q}\right)\right)=\lambda\left(\phi_{1}^{p-1-\alpha}+f\left(\bar{\phi}_{1}\right)\right), \\
-\Delta_{q} \bar{\phi}_{1} & =\lambda M_{\lambda}^{q-1} \geq \lambda\left(\lambda^{(q-1-\beta) /(q-1)} M_{\lambda}^{q-1-\beta}\left\|e_{q}\right\|_{\infty}^{q-1-\beta}+\|g\|_{\infty}\right) \\
& \geq \lambda\left(\left(\lambda^{1 /(q-1)} M_{\lambda} e_{q}\right)^{q-1-\beta}+g\left(\lambda^{1 /(p-1)} M_{\lambda} e_{p}\right)\right)=\lambda\left(\bar{\phi}_{1}^{q-1-\beta}+g\left(\phi_{1}\right)\right) .
\end{aligned}
$$

Thus, $\left(\phi_{1}, \bar{\phi}_{1}\right)$ is a supersolution of (1.1) for all $\lambda>0$.

Suppose $g(s) \rightarrow \infty$ as $s \rightarrow \infty$. Choose $M_{\lambda} \gg 1$ such that

$$
\frac{1}{\left\|e_{p}\right\|_{\infty}^{p-1}} \geq \lambda\left(\frac{1}{M_{\lambda}^{\alpha}\left\|e_{p}\right\|_{\infty}^{\alpha}}+\frac{\left.f\left((2 \lambda)^{1 /(q-1)}\left\|e_{q}\right\|_{\infty} g\left(M_{\lambda}\left\|e_{p}\right\|_{\infty}\right)^{1 /(q-1}\right)\right)}{\left(M_{\lambda}\left\|e_{p}\right\|_{\infty}\right)^{p-1}}\right)
$$


and

$$
g\left(M_{\lambda}\left\|e_{p}\right\|_{\infty}\right)^{\beta /(q-1)} \geq(2 \lambda)^{(q-1-\beta) /(q-1)}\left\|e_{q}\right\|_{\infty}^{q-1-\beta} .
$$

Let $\left(\phi_{1}, \bar{\phi}_{1}\right)=\left(M_{\lambda} e_{p},(2 \lambda)^{1 /(q-1)} g\left(M_{\lambda}\left\|e_{p}\right\|_{\infty}\right)^{1 /(q-1)} e_{q}\right)$. Then

$$
\begin{aligned}
-\Delta_{p} \phi_{1} & =M_{\lambda}^{p-1} \\
& \geq \lambda\left(M_{\lambda}^{p-1-\alpha}\left\|e_{p}\right\|_{\infty}^{p-1-\alpha}+f\left((2 \lambda)^{1 /(q-1)} g\left(M_{\lambda}\left\|e_{p}\right\|_{\infty}\right)^{1 /(q-1)}\left\|e_{q}\right\|_{\infty}\right)\right) \\
& \geq \lambda\left(\left(M_{\lambda} e_{p}\right)^{p-1-\alpha}+f\left((2 \lambda)^{1 /(q-1)} g\left(M_{\lambda}\left\|e_{p}\right\|_{\infty}\right)^{1 /(q-1)} e_{q}\right)\right) \\
& =\lambda\left(\phi_{1}^{p-1-\alpha}+f\left(\bar{\phi}_{1}\right)\right)
\end{aligned}
$$

and

$$
\begin{aligned}
& -\Delta_{q} \bar{\phi}_{1}=2 \lambda g\left(M_{\lambda}\left\|e_{p}\right\|_{\infty}\right) \\
& \quad \geq \lambda\left((2 \lambda)^{(q-1-\beta) /(q-1)} g\left(M_{\lambda}\left\|e_{p}\right\|_{\infty}\right)^{(q-1-\beta) /(q-1)}\left\|e_{q}\right\|_{\infty}^{q-1-\beta}+g\left(M_{\lambda}\left\|e_{p}\right\|_{\infty}\right)\right) \\
& \quad \geq \lambda\left(\left((2 \lambda)^{1 /(q-1)} g\left(M_{\lambda}\left\|e_{p}\right\|_{\infty}\right)^{1 /(q-1)} e_{q}\right)^{q-1-\beta}+g\left(M_{\lambda} e_{p}\right)\right) \\
& \quad=\lambda\left(\bar{\phi}_{1}^{q-1-\beta}+g\left(\phi_{1}\right)\right) .
\end{aligned}
$$

Thus, $\left(\phi_{1}, \bar{\phi}_{1}\right)$ is a supersolution of (1.1) for all $\lambda>0$. If $g$ is bounded and $f(s) \rightarrow \infty$ as $s \rightarrow \infty$, then $\lim _{s \rightarrow \infty} g\left(M f(s)^{1 /(p-1)}\right) / s^{q-1}=0$ for every $M>$ 0 . Then $\left(\phi_{1}, \bar{\phi}_{1}\right)=\left((2 \lambda)^{1 /(p-1)} f\left(M_{\lambda}\left\|e_{q}\right\|_{\infty}\right)^{1 /(p-1)} e_{p}, M_{\lambda} e_{q}\right)$ is a supersolution of (1.1) for all $\lambda>0$ by arguments similar to the previous case with the roles of $f$ and $g$ interchanged. Also, in each case if $M_{\lambda}$ is sufficiently large, $\left(\psi_{1}, \bar{\psi}_{1}\right) \leq$ $\left(\phi_{1}, \bar{\phi}_{1}\right)$. Hence, by Proposition 1.8 , there exists a positive solution $(u, v)$ such that $\left(\psi_{1}, \bar{\psi}_{1}\right) \leq(u, v) \leq\left(\phi_{1}, \bar{\phi}_{1}\right)$ for each $\lambda>0$.

Proof of Theorem 1.3. We first establish this result when $\Omega$ is a ball of radius $R$. Let $\left(\phi_{2}, \bar{\phi}_{2}\right)=\left(a_{1} e_{p} /\left\|e_{p}\right\|_{\infty}, a_{1} e_{q} /\left\|e_{q}\right\|_{\infty}\right)$. For

$$
\lambda<\min \left[\frac{1}{2\left\|e_{p}\right\|_{\infty}^{p-1}} \min \left\{a_{1}^{\alpha}, \frac{a_{1}^{p-1}}{f\left(a_{1}\right)}\right\}, \frac{1}{2\left\|e_{q}\right\|_{\infty}^{q-1}} \min \left\{a_{1}^{\beta}, \frac{a_{1}^{q-1}}{g\left(a_{1}\right)}\right\}\right],
$$

we have

$$
\begin{aligned}
-\Delta_{p} \phi_{2} & =\frac{a_{1}^{p-1}}{\left\|e_{p}\right\|_{\infty}^{p-1}}=\frac{a_{1}^{p-1}}{2\left\|e_{p}\right\|_{\infty}^{p-1}}+\frac{a_{1}^{p-1}}{2\left\|e_{p}\right\|_{\infty}^{p-1}}>\lambda a_{1}^{p-1-\alpha}+\lambda f\left(a_{1}\right) \\
& \geq \lambda\left(\left(\frac{a_{1}}{\left\|e_{p}\right\|_{\infty}} e_{p}\right)^{p-1-\alpha}+f\left(\frac{a_{1}}{\left\|e_{q}\right\|_{\infty}} e_{q}\right)\right)=\lambda\left(\phi_{2}^{p-1-\alpha}+f\left(\bar{\phi}_{2}\right)\right)
\end{aligned}
$$

and

$$
\begin{aligned}
-\Delta_{q} \bar{\phi}_{2} & =\frac{a_{1}^{q-1}}{\left\|e_{q}\right\|_{\infty}^{q-1}}=\frac{a_{1}^{q-1}}{2\left\|e_{q}\right\|_{\infty}^{q-1}}+\frac{a_{1}^{q-1}}{2\left\|e_{q}\right\|_{\infty}^{q-1}}>\lambda a_{1}^{q-1-\beta}+\lambda g\left(a_{1}\right) \\
& \geq \lambda\left(\left(\frac{a_{1}}{\left\|e_{q}\right\|_{\infty}} e_{q}\right)^{q-1-\beta}+g\left(\frac{a_{1}}{\left\|e_{p}\right\|_{\infty}} e_{p}\right)\right)=\lambda\left(\bar{\phi}_{2}^{q-1-\beta}+g\left(\phi_{2}\right)\right) .
\end{aligned}
$$

Hence $\left(\phi_{2}, \bar{\phi}_{2}\right)$ is a strict supersolution with $\left\|\phi_{2}\right\|_{\infty}=\left\|\bar{\phi}_{2}\right\|_{\infty}=a_{1}$. 
We construct a positive strict subsolution $\left(\psi_{2}, \bar{\psi}_{2}\right)$ of (1.1) for

$$
\lambda>\lambda_{*}=\min \left[\max \left\{\frac{a_{2}^{p-1}}{f\left(a_{2}\right)} A_{p, p}, \frac{a_{2}^{q-1}}{g\left(a_{2}\right)} A_{p, q}\right\}, \max \left\{\frac{a_{2}^{p-1}}{f\left(a_{2}\right)} A_{q, p}, \frac{a_{2}^{q-1}}{g\left(a_{2}\right)} A_{q, q}\right\}\right] .
$$

Consider the following boundary value problem:

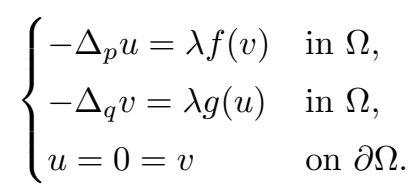

For $0<\varepsilon<R$ and $\delta, \eta>1$, define $\rho:[0, R] \rightarrow[0,1]$ by

$$
\rho(t)= \begin{cases}1 & \text { for } 0 \leq t \leq \varepsilon \\ 1-\left(1-\left(\frac{R-t}{R-\varepsilon}\right)^{\eta}\right)^{\delta} & \text { for } \varepsilon<t \leq R .\end{cases}
$$

Then

$$
\rho^{\prime}(t)= \begin{cases}0 & \text { for } 0 \leq t \leq \varepsilon \\ -\frac{\delta \eta}{R-\varepsilon}\left(\frac{R-t}{R-\varepsilon}\right)^{\eta-1}\left(1-\left(\frac{R-t}{R-\epsilon}\right)^{\eta}\right)^{\delta-1} & \text { for } \varepsilon<t \leq R .\end{cases}
$$

Let $d(t)=a_{2} \rho(t)$. Define $\left(\psi_{2}, \bar{\psi}_{2}\right)$ as the positive radially symmetric and decreasing solution of

$$
\begin{cases}-\Delta_{p} \psi_{2}=\lambda f(d) & \text { in } B_{R} \\ -\Delta_{q} \bar{\psi}_{2}=\lambda g(d) & \text { in } B_{R} \\ \psi_{2}=0=\bar{\psi}_{2} & \text { on } \partial B_{R}\end{cases}
$$

Then $\psi_{2}$ and $\bar{\psi}_{2}$ satisfy

$$
\begin{cases}-\left(t^{N-1} \varphi_{p}\left(\psi_{2}^{\prime}(t)\right)\right)^{\prime}=\lambda t^{N-1} f(d(t)) & \text { for } t \in(0, R), \\ -\left(t^{N-1} \varphi_{q}\left(\bar{\psi}_{2}^{\prime}(t)\right)\right)^{\prime}=\lambda t^{N-1} g(d(t)) & \text { for } t \in(0, R), \\ \psi_{2}^{\prime}(0)=0, \quad \psi_{2}(R)=0, \quad \bar{\psi}_{2}^{\prime}(0)=0, & \bar{\psi}_{2}(R)=0,\end{cases}
$$

where $\varphi_{m}(t)=|t|^{m-2} t$ for all $t \in \mathbb{R}$. Integrating once, we get for $0<t<R$,

$$
\begin{aligned}
& -\varphi_{p}\left(\psi_{2}^{\prime}(t)\right)=\frac{\lambda}{t^{N-1}} \int_{0}^{t} s^{N-1} f(d(s)) d s, \\
& -\varphi_{q}\left(\bar{\psi}_{2}^{\prime}(t)\right)=\frac{\lambda}{t^{N-1}} \int_{0}^{t} s^{N-1} g(d(s)) d s .
\end{aligned}
$$

Since $\varphi_{m}$ is monotone, $\varphi_{m}^{-1}$ is also continuous and monotone. Hence, we have

$$
\begin{aligned}
& -\psi_{2}^{\prime}(t)=\varphi_{p}^{-1}\left(\frac{\lambda}{t^{N-1}} \int_{0}^{t} s^{N-1} f(d(s)) d s\right), \\
& -\bar{\psi}_{2}^{\prime}(t)=\varphi_{q}^{-1}\left(\frac{\lambda}{t^{N-1}} \int_{0}^{t} s^{N-1} g(d(s)) d s\right) .
\end{aligned}
$$


Bifurcation and Multiplicity Results for Classes of $p, q$-Laplacian Systems 111

For $\lambda>\lambda_{*}$, we claim that

$$
\psi_{2}(t)>d(t) \quad \text { and } \quad \bar{\psi}_{2}(t)>d(t), \quad 0 \leq t<R .
$$

If our claim is true, $\left(\psi_{2}, \bar{\psi}_{2}\right)$ is a strict subsolution of the boundary value problem (1.1) since

$$
\begin{array}{ll}
-\Delta_{p} \psi_{2}=\lambda f(d)<\lambda\left(\psi_{2}^{p-1-\alpha}+f\left(\bar{\psi}_{2}\right)\right) & \text { in } B_{R} \\
-\Delta_{q} \bar{\psi}_{2}=\lambda g(d)<\lambda\left(\bar{\psi}_{2}^{q-1-\beta}+g\left(\psi_{2}\right)\right) & \text { in } B_{R} .
\end{array}
$$

It suffices to prove that $\psi_{2}^{\prime}(t)<d^{\prime}(t)$ and $\bar{\psi}_{2}^{\prime}(t)<d^{\prime}(t)$ on $(0, R]$ in order to show (3.1) since $\psi_{2}(R)=\bar{\psi}_{2}(R)=0=d(R)$. It is obvious on $(0, \varepsilon]$ because $\psi_{2}^{\prime}(t)<0=d^{\prime}(t)$ and $\bar{\psi}_{2}^{\prime}(t)<0=d^{\prime}(t)$. For $t>\varepsilon$, we have

$$
\begin{aligned}
-\psi_{2}^{\prime}(t) & =\varphi_{p}^{-1}\left(\frac{\lambda}{t^{N-1}} \int_{0}^{t} s^{N-1} f(d(s)) d s\right) \geq \varphi_{p}^{-1}\left(\frac{\lambda}{R^{N-1}} \int_{0}^{\varepsilon} s^{N-1} f(d(s)) d s\right) \\
& =\varphi_{p}^{-1}\left(\frac{\lambda}{R^{N-1}} \int_{0}^{\varepsilon} s^{N-1} f\left(a_{2}\right) d s\right)=\varphi_{p}^{-1}\left(\frac{\lambda}{R^{N-1}} \frac{\varepsilon^{N}}{N} f\left(a_{2}\right)\right)
\end{aligned}
$$

and

$$
\begin{aligned}
-\bar{\psi}_{2}^{\prime}(t) & =\varphi_{q}^{-1}\left(\frac{\lambda}{t^{N-1}} \int_{0}^{t} s^{N-1} g(d(s)) d s\right) \geq \varphi_{q}^{-1}\left(\frac{\lambda}{R^{N-1}} \int_{0}^{\varepsilon} s^{N-1} g(d(s)) d s\right) \\
& =\varphi_{q}^{-1}\left(\frac{\lambda}{R^{N-1}} \int_{0}^{\varepsilon} s^{N-1} g\left(a_{2}\right) d s\right)=\varphi_{q}^{-1}\left(\frac{\lambda}{R^{N-1}} \frac{\varepsilon^{N}}{N} g\left(a_{2}\right)\right) .
\end{aligned}
$$

Noting that $\left|d^{\prime}(t)\right| \leq a_{2} \delta \eta /(R-\varepsilon)$ on $(\varepsilon, R)$, it is easy to see that $\psi_{2}^{\prime}(t)<d^{\prime}(t)$ and $\bar{\psi}_{2}^{\prime}(t)<d^{\prime}(t)$ on $(\varepsilon, R)$ provided

$$
\varphi_{p}^{-1}\left(\frac{\lambda}{R^{N-1}} \frac{\varepsilon^{N}}{N} f\left(a_{2}\right)\right)>a_{2} \frac{\delta \eta}{R-\varepsilon} \quad \text { and } \quad \varphi_{q}^{-1}\left(\frac{\lambda}{R^{N-1}} \frac{\varepsilon^{N}}{N} g\left(a_{2}\right)\right)>a_{2} \frac{\delta \eta}{R-\varepsilon} .
$$

Equivalently, if

$$
\lambda>\max \left\{(\delta \eta)^{p-1} \frac{a_{2}^{p-1}}{f\left(a_{2}\right)} \frac{R^{N-1} N}{\varepsilon^{N}(R-\varepsilon)^{p-1}},(\delta \eta)^{q-1} \frac{a_{2}^{q-1}}{g\left(a_{2}\right)} \frac{R^{N-1} N}{\varepsilon^{N}(R-\varepsilon)^{q-1}}\right\} .
$$

Now, if

$$
\lambda_{*}=\max \left\{\frac{a_{2}^{p-1}}{f\left(a_{2}\right)} A_{p, p}, \frac{a_{2}^{q-1}}{g\left(a_{2}\right)} A_{p, q}\right\},
$$

choosing $\varepsilon=N R /(N+p-1)$ in the definition of $\rho$, (3.2) reduces to showing

$$
\lambda>\max \left\{(\delta \eta)^{p-1} \frac{a_{2}^{p-1}}{f\left(a_{2}\right)} A_{p, p},(\delta \eta)^{q-1} \frac{a_{2}^{q-1}}{g\left(a_{2}\right)} A_{p, q}\right\} .
$$

But $\lambda>\lambda_{*}$. Hence we can choose $\delta(>1)$ and $\eta(>1)$ such that (3.3) is satisfied. Next, if

$$
\lambda_{*}=\max \left\{\frac{a_{2}^{p-1}}{f\left(a_{2}\right)} A_{q, p}, \frac{a_{2}^{q-1}}{g\left(a_{2}\right)} A_{q, q}\right\},
$$


choosing $\varepsilon=N R /(N+q-1)$ in the definition of $\rho,(3.2)$ reduces to showing

$$
\lambda>\max \left\{(\delta \eta)^{p-1} \frac{a_{2}^{p-1}}{f\left(a_{2}\right)} A_{q, p},(\delta \eta)^{q-1} \frac{a_{2}^{q-1}}{g\left(a_{2}\right)} A_{q, q}\right\} .
$$

Again, since $\lambda>\lambda_{*}$, we can choose $\delta(>1)$ and $\eta(>1)$ such that (3.4) is satisfied. Hence, (3.1) holds for $\lambda>\lambda_{*}$. Thus, $\left(\psi_{2}, \bar{\psi}_{2}\right)$ is a strict subsolution of (1.1). From the proof of Theorem 1.2, we have a sufficiently small positive subsolution $\left(\psi_{1}, \bar{\psi}_{1}\right)$ and a sufficiently large positive supersolution $\left(\phi_{1}, \bar{\phi}_{1}\right)$ such that $\left(\psi_{1}, \bar{\psi}_{1}\right) \leq\left(\phi_{2}, \bar{\phi}_{2}\right) \leq\left(\phi_{1}, \bar{\phi}_{1}\right)$ and $\left(\psi_{1}, \bar{\psi}_{1}\right) \leq\left(\psi_{2}, \bar{\psi}_{2}\right) \leq\left(\phi_{1}, \bar{\phi}_{1}\right)$. Since $\left\|\psi_{2}\right\|_{\infty} \geq\|d\|_{\infty}=a_{2}$ and $\left\|\phi_{2}\right\|_{\infty}=a_{1}$, we have $\left(\psi_{2}, \bar{\psi}_{2}\right) \not \leq\left(\phi_{2}, \bar{\phi}_{2}\right)$. By Proposition 1.9, (1.1) has at least three distinct solutions for $\lambda \in\left(\lambda_{*}, \lambda^{*}\right)$.

Next, when $\Omega$ is a general bounded domain, let $B_{R}$ be the largest inscribed ball in $\Omega$. Define

$$
\chi(x)=\left\{\begin{array}{ll}
\psi_{2} & \text { for } x \in B_{R}, \\
0 & \text { for } x \in \Omega-B_{R},
\end{array} \text { and } \bar{\chi}(x)= \begin{cases}\bar{\psi}_{2} & \text { for } x \in B_{R}, \\
0 & \text { for } x \in \Omega-B_{R},\end{cases}\right.
$$

where $\left(\psi_{2}, \bar{\psi}_{2}\right)$ is a second subsolution of (1.1) constructed above for $\Omega=B_{R}$. Then $\chi \in W^{1, p}(\Omega) \cap C(\bar{\Omega})$ and $\bar{\chi} \in W^{1, q}(\Omega) \cap C(\bar{\Omega})$. Further, on $B_{R}$ we have

$$
\begin{aligned}
& -\Delta_{p} \chi=-\Delta_{p} \psi_{2}<\lambda\left(\psi_{2}^{p-1-\alpha}+f\left(\bar{\psi}_{2}\right)\right)=\lambda\left(\chi^{p-1-\alpha}+f(\bar{\chi})\right), \\
& -\Delta_{q} \bar{\chi}=-\Delta_{q} \bar{\psi}_{2}<\lambda\left(\bar{\psi}_{2}^{q-1-\beta}+g\left(\psi_{2}\right)\right)=\lambda\left(\bar{\chi}^{q-1-\beta}+g(\chi)\right),
\end{aligned}
$$

while outside $B_{R}$ we have

$$
-\Delta_{p} \chi=0=\lambda\left(\chi^{p-1-\alpha}+f(\bar{\chi})\right) \text { and } \quad-\Delta_{q} \bar{\chi}=0=\lambda\left(\bar{\chi}^{q-1-\beta}+g(\chi)\right) .
$$

Hence, $(\chi, \bar{\chi})$ is a strict subsolution of (1.1) in $\Omega$. The rest of the proof is identical to the previous case except that here for the second subsolution we will use $(\chi, \bar{\chi})$ described above.

\section{Examples}

We illustrate in this section simple examples that satisfy the hypotheses in Theorems 1.3 and 1.6.

EXAMPLE 4.1. Consider the boundary value problem

$$
\begin{cases}-\Delta_{p} u=\lambda\left\{u^{p-1-\alpha}+e^{\tau u /(\tau+u)}-1\right\} & \text { in } \Omega, \\ u=0 & \text { on } \partial \Omega,\end{cases}
$$

where $f(s)=e^{\tau s /(\tau+s)}-1$ with $\tau>0$ and $\Omega$ is a bounded domain in $\mathbb{R}^{N}$ with smooth boundary $\partial \Omega$. Clearly, $f(0)=0$, and $f(s) / s^{p-1} \rightarrow 0$ as $s \rightarrow \infty$ since $f$ is bounded for each $\tau>0$. 
Next, choosing $a_{1}=1$ and $a_{2}=\tau$, we have

$$
A=\frac{\min \left\{a_{1}^{\alpha}, a_{1}^{p-1} / f\left(a_{1}\right)\right\}}{a_{2}^{p-1} / f\left(a_{2}\right)}=\frac{1}{\tau^{p-1}} \frac{e^{\tau / 2}-1}{e^{\tau /(1+\tau)}-1} \text { for } \tau \gg 1
$$

and hence $A \rightarrow \infty$ as $\tau \rightarrow \infty$. Hence, the hypotheses in Theorem 1.6 are satisfied. In particular, for any $\tau>0,(4.1)$ has a positive solution for all $\lambda>0$, and for sufficiently large $\tau,(4.1)$ has at least three positive solutions for $\lambda \in\left(\lambda_{*}, \lambda^{*}\right)$ where

$$
\lambda_{*}=\frac{\tau^{p-1}}{e^{\tau / 2}-1} A_{p, p} \quad \text { and } \quad \lambda^{*}=\frac{1}{2\left\|e_{p}\right\|_{\infty}^{p-1}\left(e^{\tau /(1+\tau)}-1\right)} .
$$

In fact, given $\lambda \in\left(0,1 /\left(2\left\|e_{p}\right\|_{\infty}^{p-1}(e-1)\right)\right)$, there exists $\tau_{0}>0$ such that $(4.1)$ has at least three positive solutions for $\tau>\tau_{0}$.

EXAmple 4.2. Consider the system

$$
\begin{cases}-\Delta_{p} u=\lambda\left\{u^{p-1-\alpha}+e^{\tau v /(\tau+v)}-1\right\} & \text { in } \Omega, \\ -\Delta_{q} v=\lambda\left\{v^{q-1-\beta}+u^{\xi}\right\} & \text { in } \Omega, \\ u=0=v & \text { on } \partial \Omega,\end{cases}
$$

where $f(s)=e^{\tau s /(\tau+s)}-1$ with $\tau>0, g(s)=s^{\xi}$ with $\xi>0$ and $\Omega$ is a bounded domain in $\mathbb{R}^{N}$ with smooth boundary $\partial \Omega$. Clearly, $f(0)=g(0)=0$ and $\left(\mathrm{H}_{1}\right)$ is satisfied since $f$ is bounded for each $\tau>0$. Hence, Theorems 1.1 and 1.2 hold for all $\tau>0$ and $\xi>0$. Next, choosing $a_{1}=1$ and $a_{2}=\tau$,

$$
A=\frac{\min \left[\min \left\{a_{1}^{\alpha}, a_{1}^{p-1} / f\left(a_{1}\right)\right\}, \min \left\{a_{1}^{\beta}, a_{1}^{q-1} / g\left(a_{1}\right)\right\}\right]}{\max \left\{a_{2}^{p-1} / f\left(a_{2}\right), a_{2}^{q-1} / g\left(a_{2}\right)\right\}}=\frac{\tau^{\xi-q+1}}{e^{\tau /(1+\tau)}-1} \quad \text { for } \tau \gg 1
$$

and hence $\left(\mathrm{H}_{2}\right)$ is satisfied for $\tau \gg 1$ and $\xi>q-1$ since $A \rightarrow \infty$ as $\tau \rightarrow \infty$. For sufficiently large $\tau$, there exist at least three positive solutions of (4.2) for $\lambda \in\left(\lambda_{*}, \lambda^{*}\right)$ where $\lambda_{*}=\tau^{q-1-\xi} \min \left\{A_{p, q}, A_{q, q}\right\}$ and

$$
\lambda^{*}=\min \left\{\frac{1}{2\left\|e_{p}\right\|_{\infty}^{p-1}\left(e^{\tau /(1+\tau)}-1\right)}, \frac{1}{2\left\|e_{q}\right\|_{\infty}^{q-1}}\right\} .
$$

In fact, given

$$
\lambda \in\left(0, \min \left\{\frac{1}{2\left\|e_{p}\right\|_{\infty}^{p-1}(e-1)}, \frac{1}{2\left\|e_{q}\right\|_{\infty}^{q-1}}\right\}\right),
$$

there exists $\tau_{0}>0$ such that (4.2) has at least three positive solutions for $\tau>\tau_{0}$.

\section{REFERENCES}

[1] J. Ali, K.J. Brown and R. ShivaJi, Positive solutions for $n \times n$ elliptic systems with combined nonlinear effects, Differential Integral Equations 24 (2011), No. 3-4, 307-324.

[2] J. Ali And R. ShIVAJI, Multiple positive solutions for a class of p-q-Laplacian systems with multiple parameters and combined nonlinear effects, Differential Integral Equations 22 (2009), No. 7-8, 669-678. 
[3] H. Amann, Fixed point equations and nonlinear eigenvalue problems in ordered Banach spaces, SIAM Rev. 18 (1976), 620-709.

[4] M. Belloni And B. Kawohl, A direct uniqueness proof for equations involving the $p$ Laplace operator, Manuscripta Math. 109 (2002), 229-231.

[5] K.J. Brown, M.M.A. Ibrahim and R. Shivaji, S-shaped bifurcation curves, Nonlinear Anal. 5 (1981), No. 5, 475-486.

[6] C. Maya, S. Oruganti and R. Shivaji, Positive solutions for classes of p-Laplacian equations, Differential Integral Equations 16 (2003), No. 6, 757-768.

[7] M. Ramaswamy and R. Shivaji, Multiple positive solutions for classes of p-Laplacian equations, Differential Integral Equations 17 (2004), No. 11-12, 1255-1261.

[8] R. Shivaji, A remark on the existence of three solutions via sub-super solutions, Nonlinear Analysis and Application, Lecture Notes in Pure and Applied Mathematics (V. Lakshmikantham, ed.) 109 (1987), 561-566.

Manuscript received December 29, 2014 accepted March 17, 2015

Ratnasingham Shivaji and Byungjae Son

Department of Mathematics and Statistics

University of North Carolina at Greensboro

Greensboro, NC 27412, USA

E-mail address: shivaji@uncg.edu,b_son@uncg.edu 